\title{
Cluster ion beam assisted fabrication of metallic nanostructures for plasmonic applications
}

\author{
Iram Saleem $^{\text {a,* }}$, Buddhi P Tilakaratne ${ }^{\text {a,b }}$, Yang Li ${ }^{c}$, Jiming Bao ${ }^{c}$, Dharshana N Wijesundera ${ }^{a}$ \\ and Wei-Kan Chu ${ }^{\text {a }}$ \\ ${ }^{a}$ Department of Physics and Texas Center for Superconductivity, University of Houston, Houston, \\ TX, 77204, USA \\ ${ }^{b}$ Currently at Department of Radiology, Pennsylvania State University, University Park, \\ Philadelphia, PA, 16801, USA. \\ ${ }^{c}$ Department of Electrical and Computer Engineering, University of Houston, Houston, TX, 77204, \\ $U S A$
}

*Corresponding author email address: iiram.qau@gmail.com, isaleem@uh.edu

\begin{abstract}
We report a high-throughput, single-step method for fabricating rippled plasmonic nanostructure arrays via self-assembly induced by oblique angle cluster ion beam irradiation of metal surfaces. This approach does not require lithographic or chemical processes and has the prominent advantage of possible large surface area coverage and applicability to different starting materials. The polarization dependent plasmonic property of the gold nano-ripple is due to their one dimension structure. The localized plasmon resonance frequency of synthesized nano-ripple arrays is tunable by changing nano-ripple dimensions that can be engineered by changing the cluster ion beam irradiation parameters. In this specific case presented, using 30keV Ar-gas cluster ion beam, we fabricate gold nano-ripple arrays that show localized plasmon resonance in the visible range through near IR range, tunable by varying cluster ion irradiation fluence.
\end{abstract}

Keywords: localized surface plasmon resonance, Ion beam nano-fabrication, cluster ion beam selfassembly, nano ripple array

\section{Introduction}

Localized surface plasmon resonance (LSPR) effect of metal nanostructures has attracted considerable research interest because of its potential applications in several areas including sensors [1], surface enhanced spectroscopy [2, 3], photovoltaics [4, 5], sub-wavelength microscopy [6, 7], near-field lithography [8], nano-photonics [9], bio-imaging [10], laser photo thermal therapy [11], and numerous other applications. LSPR results from coupled oscillations between the charge density of conduction electrons and their corresponding electromagnetic field in conductive nanostructures that are smaller in dimension compared to the wavelength of exciting electromagnetic radiation, which results in enhanced absorption and scattering resonances [12]. The unique plasmonic property of metals resides in the fact that their dielectric constant is a complex function of the frequency of incident light, $\varepsilon(\omega)=\varepsilon_{1}(\omega)+$ $i \varepsilon_{2}(\omega)$. The imaginary component $\varepsilon_{2}(\omega)$ causes the resonant behavior. The LSPR peak relies greatly on the shape, size and composition of the nanostructures. Application of the LSPR effect therefore critically depends on reliable and efficient ways of controlled fabrication of metallic nanostructures. 
Numerous nano-fabrication techniques are utilized in synthesizing nanostructures for utilization of the LSPR effect [13]. Wet chemical reduction is an attractive method for synthesizing noble metallic (Au and $\mathrm{Ag}$ ) nano particles of dimensions required for LSPR applications. Because of the face centered cubic crystallization of many metals, in this approach, the nano particles inherently crystalize into a truncated octahedron shape. However, surfactants and stabilizing agents added to the reactants allow engineering of different shapes and morphology by promoting preferential growth along certain crystal facets [14]. Wet chemical reduction synthesis has the advantage of large scale, relatively rapid synthesis. However, it is impaired in reproducibility and ability to fabricate substrates with immobilized ordered nanostructure arrangements. Another attractive approach is to promote plasmonic metal nano-particle growth in a solid solution, where the metal is infused into a solid substrate by means of ion implantation as to reach a concentration exceeding the solid solubility of the metal in the substrate [15, 16]. Nano-particles formed in this process are inherently immobilized in the solid substrate with high spatial propinquity and therefore, these composites are also effective as surface enhanced Raman spectroscopy (SERS) substrates [17].

Lithographic techniques are attractive in achieving size and shape control of plasmonic nanostructures. Fabrication of nano structures via electron beam (EB) lithography and focused ion beam lithography allows high resolution and precise control of size, shape, and spatial distribution of nano structures [13, 18, 19]. Despite their advantages, they suffer from being slow processes and are limited to relatively small area fabrication [18]. Nano sphere lithography offers a cost effective method to produce large area 2D periodic arrays of plasmonic nano structures $[20,21]$ that are also effective SERS substrates. Alterations to fabrication methodology of nano sphere lithography allows fabrication of nano structure morphologies that include nano disks, nano rings, nano holes, and even cup-like structures [22]. Nanoimprint lithography can be considered to be a next generation lithographic technique that offers high precision comparable to EB lithography, while offering high throughput suitable for large-scale patterning $[13,18]$. This method allows finest lithographic resolutions, however, limited by the resolution of mask fabrication [13]. Some attractive and effective alternate approaches include the fabrication of metallic plasmonic resonators by nano skiving of chemically synthesized micro-plates of gold [23, 24]. In this approach, microplates of metal (gold) of nano-scale thickness are embedded in epoxy, and then sliced along with epoxy via nano skiving. This forms nano wires of well-defined dimensions that act as plasmonic resonators and plasmonic waveguides. One attractive feature is that when deposited microplates are single crystalline, the structures themselves become single crystalline, making them lowloss plasmonic resonators. The approach however is a multiple step process and is unable to produce large area nano structure arrays.

Systematic laboratory procedures with cleanroom techniques do produce effective plasmonic structures, however, industrial applications such as large-area photovoltaic module production requires inexpensive and scalable techniques for controlled fabrication of metal nano patterns [25]. Simple methods to achieve this include thermal evaporation of a thin metal film on to a substrate and heating at a moderate temperature $\left(200-300{ }^{\circ} \mathrm{C}\right)$. This causes agglomeration of the metal film by surface tension that forms a random array of nanoparticles with more regularity achievable by evaporation through a porous membrane (for example porous alumina) [25-27].

To compliment such large-area high-throughput fabrication methods, in this article we discuss a method to fabricate plasmonic nano-arrays by oblique angle gas cluster ion beam (GCIB) irradiation of gold surfaces. Our method is a single step process with high throughput [28] and the capability to produce large-area fabrication (200 mm wafer processing with commercial cluster ion implanters). Further, as any ion irradiation process this method produces minimum contamination since it does not involve any 
chemical processes. The fabrication method is further reproducible, and can be applied to many different starting materials that include metals and non-metals [29-31].

Cluster ions are charged, bound units of thousands of atoms. Energetic flux of cluster ions makes a cluster ion beam. Interaction of cluster ions with a solid is significantly different from how monomer ions interact with a solid. This is because of comparatively very low kinetic energy per constituent atom of a cluster ion and the synergetic reaction caused by the simultaneous arrival of thousands of constituent atoms of a cluster at the target with high spatiotemporal propinquity [32,33]. The resulting nature of cluster ion-solid reactions is ideal for controlled surface modification. Gas cluster ion irradiation at normal incidence to a surface is widely used in research and industry for atomic scale smoothing of surfaces $[32,34,35]$. In contrast, GCIB irradiation of surfaces at an oblique angle roughens a surface, under proper conditions, producing self-assembled periodic nano-scale ripple arrays that closely resemble Aeolian sand-ripples [29-31]. Our approach utilizes this self-assembly process in order to fabricate metallic plasmonic nano structure arrays. Interestingly, with our approach, it is straightforward to control the scale and the separation of the nano-ripple structures, for example in this case of gold, to be in the range that exhibit LSPR in the visible range. In our experiment we irradiate a thin plain gold surface with ionized clusters of argon gas. Argon being an inert gas does not react with the surface and forms a one dimensional gold nano-ripple pattern due to sputtering of the gold atoms at the surface [30]. The nanoripple wavelength depends on the number of clusters hitting the surface per unit area (centimeter square). By varying fluence of these clusters we obtain nano patterns of different sizes. In this paper we have reported the variation in the localized surface plasmon resonance frequency with the change in dimension of the gold nano-ripples and the polarization dependence of the plasmonic response of these nano-ripples on the incident electric field vector due to their one dimensional structure.

\section{Experimental Section}

\subsection{Fabrication Process}

We fabricate nano-ripple structures on gold surfaces (100 nm thick gold film on silicon obtained from Sigma Aldrich) by means of oblique angle irradiation (see Figure 1) by $30 \mathrm{keV}$ Ar GCIB (with 3000 mean $\mathrm{Ar}$ atoms per cluster ion) using an Epion cluster ion implanter. In order to control the geometry of the nano-ripple structures for the purpose of tuning the LSPR resonance frequency, we change the GCIB irradiation fluence as the variable parameter. For the cases discussed, at a base angle of incidence of 60 degrees, we irradiate the gold surface with different GCIB fluence of $1 \times 10^{16}$ clusters $/ \mathrm{cm}^{2}, 2 \times 10^{16}$ clusters $/ \mathrm{cm}^{2}$ and $4 \times 10^{16}$ clusters $/ \mathrm{cm}^{2}$. The cluster ion beam flux is approximately $3.9 \times 10^{12}$ clusters $/ \mathrm{cm}^{2} / \mathrm{sec}$ and is kept constant. The variation of irradiation fluence affects the self-assembly process such that geometric parameters of the ripple arrays vary, which subsequently determines the LSPR resonance frequency. We have chosen the mentioned incident angle because it is within the ideal range of irradiation angles for the nano-ripple formation process [30]. The same holds true in our choice of the range of irradiation fluence, for example, below our choice of the lowest fluence of $1 \times 10^{16} \mathrm{clusters} / \mathrm{cm}^{2}$ nano-arrays lose their periodicity. At the fluence of $4 \times 10^{16}$ clusters $/ \mathrm{cm}^{2}$ the separation between the individual nano ripple saturates. As we increase the fluence beyond this point, height of the ripples starts reducing and an increase in the fluence does not increase the plasmon resonance frequency. The detailed mechanism of GCIB induced ripple formation and the reasons for these lower and upper bounds are explained in detail by Tilakaratne et al. in reference [30]. 


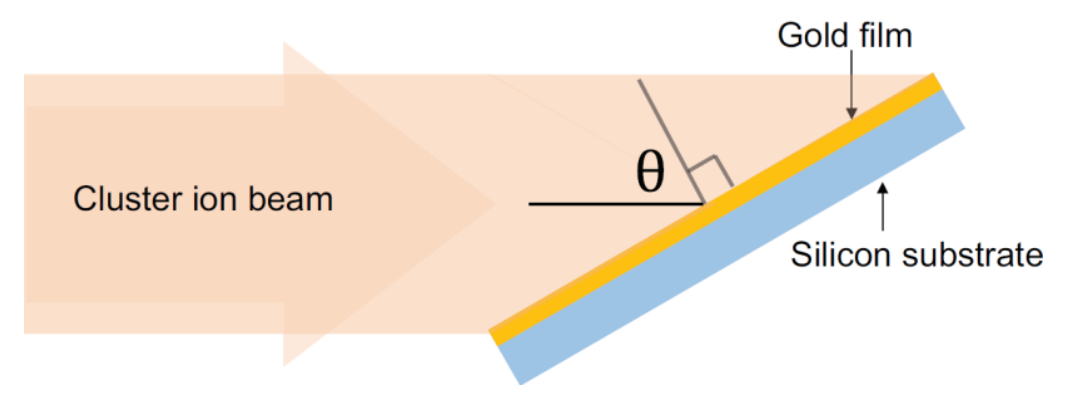

Figure 1: A schematic of oblique angle gas cluster ion beam (GCIB) irradiation configuration used in fabrication of nano ripple array structures. In this case presented, a $100 \mathrm{~nm}$ thick film of gold on silicon is irradiated with argon GCIB. $\theta$ is the angle of incidence of GCIB irradiation.

Figure $2 \mathrm{a}$ and $2 \mathrm{~b}$ show scanning electron microscope (SEM) images of the nano-ripple arrays fabricated under selected conditions of $4 \times 10^{16}$ clusters $/ \mathrm{cm}^{2}$ GCIB fluence, and 60 degrees GCIB incident angle. Tilted view in figure $2 \mathrm{~b}$ shows that the cross-section of ripples are shaped up as curved walls forming a cavity between one side of the wall and the gold substrate. Rutherford backscattering measurements of the average thickness of the remaining gold layer confirmed that silicon substrate is not exposed after irradiation. Detailed description of gold nano-rippled surfaces that cover the rest of the fabrication conditions discussed in this article can be found in references [30] and [36]. Here, we discuss LSPR of these nano-ripple structures relating it to the geometry of the rippled surfaces that depends on the fabrication conditions. As parameters that characterize the geometry, we use the ripple height (h), and the ripple periodicity (1) (average peak to peak distance of the periodic ripples) as acquired by atomic force microscopy.

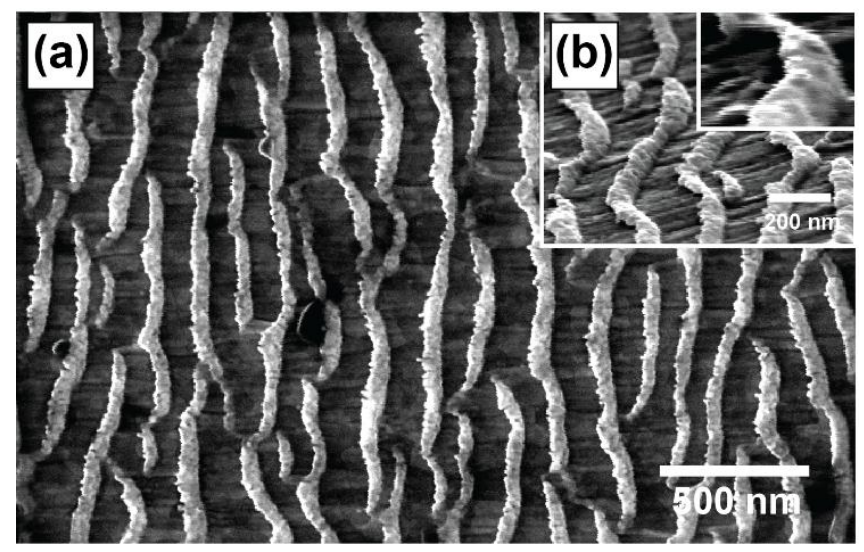

Figure 2: (a) SEM top view image of the nano-ripple array on gold surface obtained for GCIB irradiation fluence of $4 \times 10^{16}$ clusters $/ \mathrm{cm}^{2}$, for an incident angle of 60 degrees. (b) SEM image with the substrate tilted 70 degrees for better visualization of the cross-section of the ripples.

\subsection{Optical Characterization and LSPR}

We will briefly discuss the localized surface plasmon resonance (LSPR) effect from the gold nano ripple pattern. When white light falls on a plain metal surface, at a particular frequency, the electric field $\mathrm{E}$ of the incident beam and the electric field of the free conduction electrons inside the metal couple with each other. The coupling gives rise to huge charge density fluctuations which travel along the metal-dielectric interface. The quanta of these oscillations is called the surface plasmon. When the dimension of the metal is reduced to nanoscale and the wavelength of the incident light is larger than its dimension, the free electrons inside the metal nano particle show plasmonic oscillation at the resonant frequency under the 
influence of the incident electric field and give rise to standing waves due to its confined size. These are confined surface plasmon and this effect is called localized surface plasmon resonance (LSPR). However the structure under discussion is not a nano particle. It is a one dimension nano ripple pattern of gold. Thus by changing the polarization of the incident electric field on these structures the intensity of the LSPR peak will vary which explains the polarization dependence of their plasmonic behavior.

In obtaining optical spectra, we illuminate the nano-rippled surfaces with linearly polarized, focused, white light from a high-intensity tungsten light source as shown in figure 3 . The incident beam of light is perpendicular to the metal surface with the polarization perpendicular to the length (larger dimension) of nano-ripples. Diffuse scattered light off the surface is acquired and guided through an optical fiber and analyzed using an optical spectrometer.

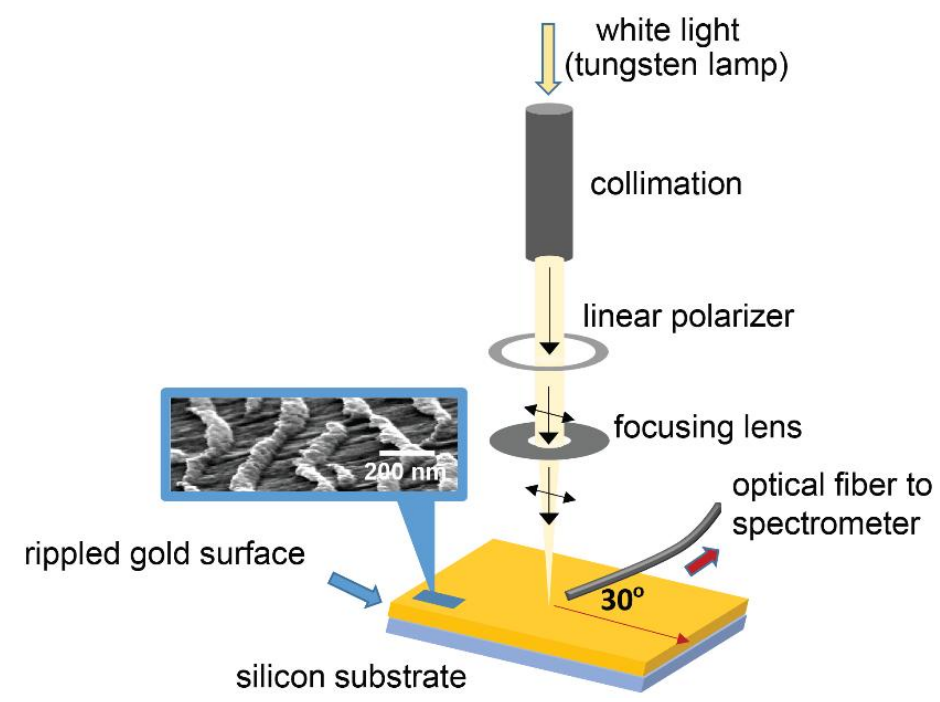

Figure 3: Experimental arrangement used for measuring optical diffuse scattering from nano-rippled gold substrates.

Figure 4a shows optical scattering spectra of rippled surfaces fabricated by GCIB irradiation at fluence of $1 \times 10^{16}$ clusters $/ \mathrm{cm}^{2}, 2 \times 10^{16}$ clusters $/ \mathrm{cm}^{2}$, and $4 \times 10^{16}$ clusters $/ \mathrm{cm}^{2}$, for GCIB incident angle of 60 degrees. The enhanced optical scattering peak caused by LSPR of the nano-ripple structures is prominent in these spectra. Figure $4 \mathrm{~b}$ and $4 \mathrm{c}$ show the variations of nano-ripple height and ripple periodicity that correspond to the peaks of the scattering spectra. The LSPR scattering enhancement is observed when the surface component of the polarization of incident light is perpendicular to the length of the ripple structures, and is suppressed when the polarization is parallel to it as is indicated in the insert of figure 4a. The suppression as expected occurs due to the electric vector of the incident beam being parallel to the length of the ripple structure that is significantly larger in dimension than the width. 

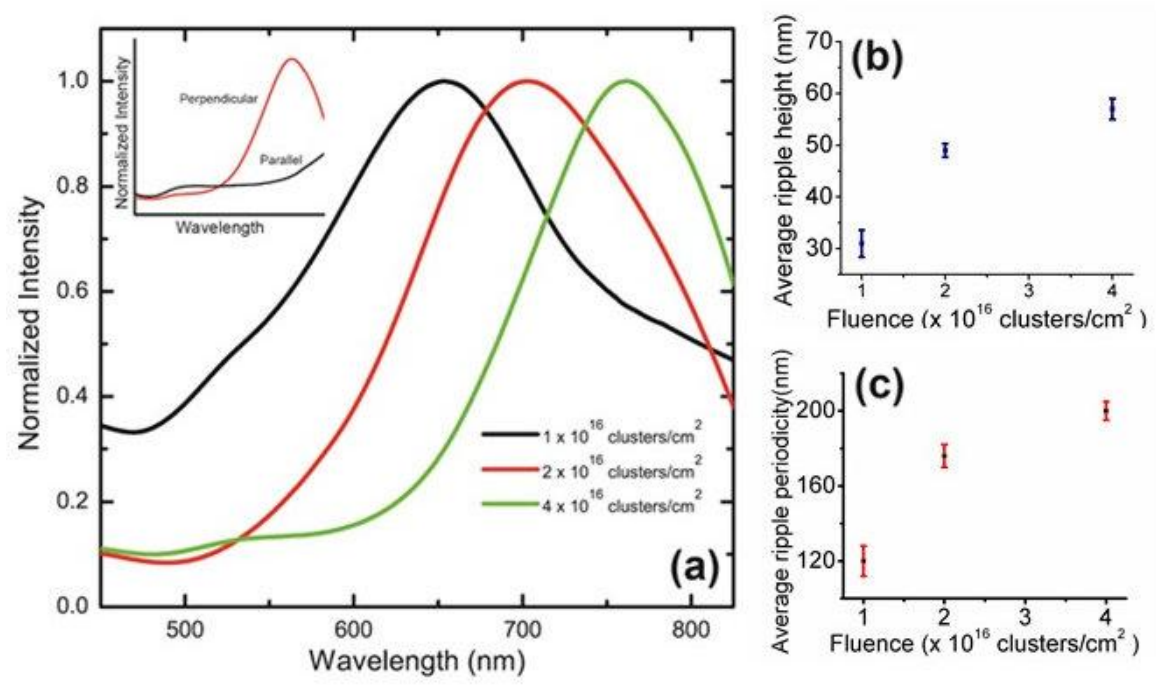

Figure 4: (a) Optical scattering spectra of gold nano-ripple array surfaces fabricated by GCIB irradiation at fluence of $1 \times 10^{16}$ clusters $/ \mathrm{cm}^{2}, 2 \times 10^{16}$ clusters $/ \mathrm{cm}^{2}$, and $4 \times 10^{16}$ clusters $/ \mathrm{cm}^{2}$, for a GCIB incident angle of 60 degrees. Spectra clearly show the enhanced scattering peak due to LSPR of the nano-ripples. The insert shows a comparison of spectra for surface component of polarization of light perpendicular and parallel to the length of ripples showing that LSPR occurs when the polarization is perpendicular to the length of ripples. The frequency of resonance can be effectively changed by changing the GCIB irradiation fluence in fabrication. (b) Variation of average ripple height and (c) average ripple periodicity with respect to GCIB fluence, obtained via atomic force microscopy.

AFM images of these nano ripple structures are shown in figure 5 and the process of ripple formation is explained in detail in reference [36]. By changing the irradiation fluence in fabrication (for 60 degree GCIB incident angle), the LSPR wavelength in the nano-ripple surfaces can be changed in a considerable range of $653 \mathrm{~nm}$ to $761 \mathrm{~nm}$. Certainly, although we have limited the selection of fluence used for the purpose of this study that is primarily a proof of concept, larger control can be achieved by a much finer selection of irradiation fluence. In addition, the dimensions of the nano ripple structures can be tailored by changing the GCIB angle of incidence [29] as an additional potential parameter for LSPR control.

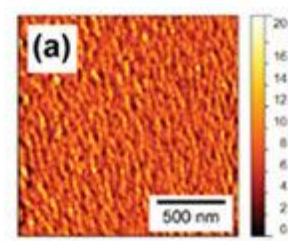

$1 \times 10^{15}$ clusters $/ \mathrm{cm}^{2}$

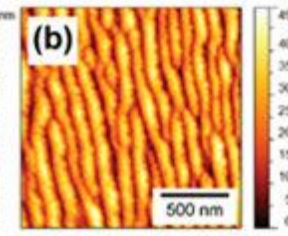

$1 \times 10^{16}$ clusters $/ \mathrm{cm}^{2}$

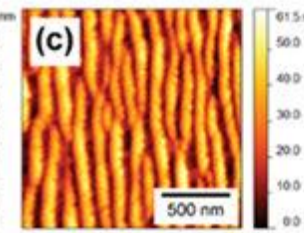

$2 \times 10^{16}$ clusters $/ \mathrm{cm}^{2}$

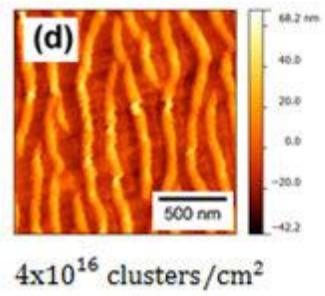

$4 \times 10^{16}$ clusters $/ \mathrm{cm}^{2}$

Figure 5: (a) (b) (c) and (d) are the AFM images of gold nano-ripples fabricated by ion fluence of $1 \times 10^{15}$ clusters $/ \mathrm{cm}^{2}, 1 \times 10^{16}$ clusters $/ \mathrm{cm}^{2}, 2 \times 10^{16}$ clusters $/ \mathrm{cm}^{2}$ and $4 \times 10^{16}$ clusters $/ \mathrm{cm}^{2}$ respectively.

As mentioned earlier the shift of the LSPR resonance wavelength is caused by the variation of the geometry of the ripple arrays. In case of molding of ripple geometry by varying the GCIB irradiation 
fluence, it is notable (see Figure $4 \mathrm{~b}$ and $4 \mathrm{c}$ ) that the average ripple periodicity increases with increasing irradiation fluence, in this case from $120 \mathrm{~nm}$ to $200 \mathrm{~nm}$ when the fluence is increased from $1 \times 10^{16}$ clusters $/ \mathrm{cm}^{2}$ to $4 \times 10^{16}$ clusters $/ \mathrm{cm}^{2}$. Along with that, in the same range of fluence the average ripple height varies between a minimum of $30 \mathrm{~nm}$ and $60 \mathrm{~nm}$. The red-shift of the LSPR resonance frequency can be qualitatively described as being caused by the increase of ripple height perturbed by increase of ripple periodicity within this range of fluence. However, the complex cross-section shape of the ripples, including the curvature and the cavity that is observed in SEM imaging (Figure 2) are not quantified in this work and may affect the LSPR resonance frequency. Therefore, we have not yet established a functional relationship between the GCIB irradiation conditions, ripple dimensions and the SPR resonance wavelength in this article, certainly further analysis is needed in developing such a relationship. The SPR resonance wavelength is however, reproducible with the same GCIB irradiation conditions.

(a)

(b)

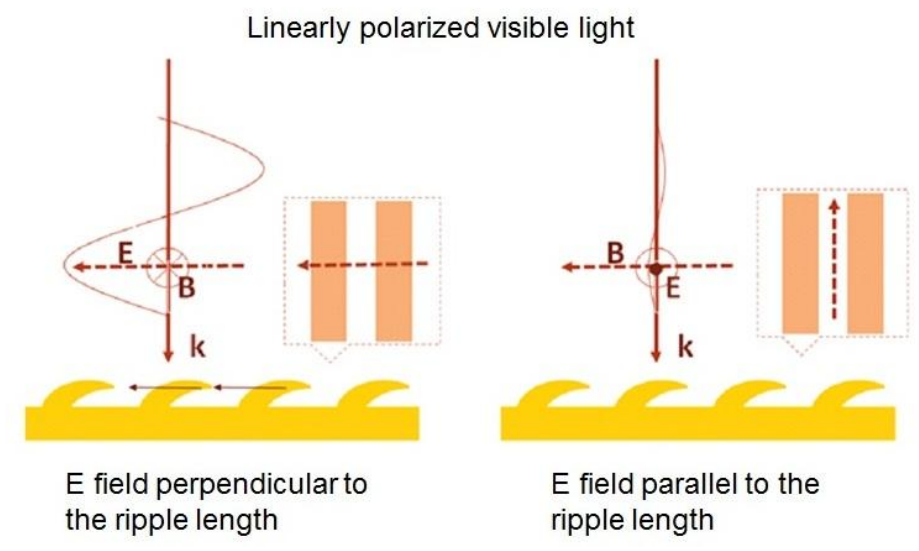

(c)

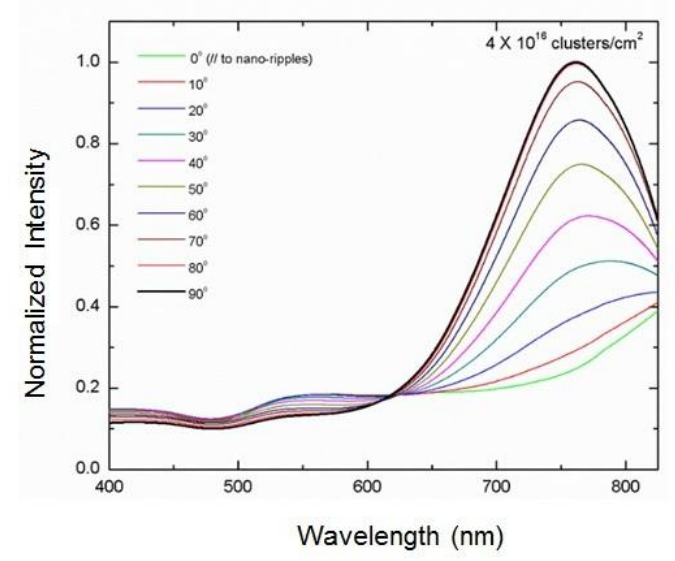

Figure 6: The polarization dependence of the plasmonic property of the nano ripples array surface is addressed. (a) Explains the LSPR effect occurs when the incident E field is perpendicular to the ripple array. That is when the wavelength of the incident $E$ field is larger than the cross section of the ripples. In the scattering spectrum huge peak in the intensity at the resonance frequency is observed. (b) Shows there is no resonance effect when the incident E field is parallel to the ripple pattern. (c) Shows the drop in the intensity as we change the polarization angle from 90 degrees to 0 degrees for the nanostructure of $4 \times 10^{16}$ clusters $/ \mathrm{cm}^{2}$ fluence. 
The intensity at the resonance wavelength varies with the polarization of the incident electric field vector, Figure 6. The intensity is highest when the ripple pattern is perpendicular to the incident E field. For LSPR to occur, the wavelength of the beam must be larger than the dimension of the ripples.

\section{Results and Discussions}

We did a comparative study of the plasmonic properties of the nano-ripple array generated by three different fluence of the cluster ion beam with intensities $1 \times 10^{16}$ clusters $/ \mathrm{cm}^{2}, 2 \times 10^{16}$ clusters $/ \mathrm{cm}^{2}$ and $4 \times 10^{16}$ clusters $/ \mathrm{cm}^{2}$. Below the fluence of $1 \times 10^{16}$ clusters $/ \mathrm{cm}^{2}$, the surface did not establish periodicity in its pattern. As we increase the fluence the structure starts to attain order. At the fluence of $1 \times 10^{16}$ clusters $/ \mathrm{cm}^{2}$, we observe the LSPR peak in the spectrum but the spectral curve is broad. The broadening of the scattering spectrum indicates that the structure is not well-ordered and thus the LSPR peak is not very sharp. We use this fluence as the lower bound for our studies. As the fluence increases the structures begin to become larger and periodic. At $2 \times 10^{16}$ clusters $/ \mathrm{cm}^{2}$ and $4 \times 10^{16}$ clusters $/ \mathrm{cm}^{2}$ we achieve a relatively uniform and ordered nano ripple pattern. As can be seen in the AFM image in Figure 5.

The scattering spectrum is narrower for the fluence of $2 \times 10^{16}$ clusters $/ \mathrm{cm}^{2}$ and becomes sharper when we increase to $4 \times 10^{16}$ clusters $/ \mathrm{cm}^{2}$ as the structure become larger and uniform with the increased fluence. The plasmon resonance frequency is different for different dimensions of the ripple patterns. The experiment indicates that as the structure gets bigger, the corresponding resonant frequency gets larger.

We plot the normalized intensity as a function of LSPR wavelength for the three different fluence in Figure 4 . The peak for the smallest fluence occurs at $653 \mathrm{~nm}$ (black line), the medium fluence (red) at 700 $\mathrm{nm}$ and the highest fluence (green) at $762 \mathrm{~nm}$ showing the relation between the LSPR peak and the nano ripple dimension. The increase in the fluence from $1 \times 10^{16}$ clusters $/ \mathrm{cm}^{2}$ to $4 \times 10^{16}$ clusters $/ \mathrm{cm}^{2}$ increases the size and the separation between the nano ripples and the corresponding LSPR wavelength from these structures increases accordingly.

The plasmonic response of the nano-ripple structure is polarization dependent because of their one dimensional nature unlike the nanoparticles. When the incident light is perpendicular to the structures we observe the maximum intensity in the LSPR resonance peak (Figure 6a). Changing the polarization will decrease the intensity gradually (Figure $6 \mathrm{c}$ ) and with E field parallel to these nano structures we observe no resonance at all (Figure $6 b$ ).

\section{Conclusions}

We have succeeded in introducing a method to fabricate metallic nano-ripple array structures by GCIB assisted self-assembly that shows prominent localized plasmon resonance. The nano-structure dimensions and therefore the corresponding resonance frequency are adjustable by changing the GCIB irradiation fluence. Although the presented case discusses nano-ripple array fabrication on a gold surface, it can very well be adopted for many different starting materials. The outcome, along with this single step, cost effective, large area GCIB based fabrication method is promising for the use of these nano ripple arrays in plasmonic applications.

\section{Acknowledgments}

This work was funded by the state of Texas through the Texas Center for Superconductivity at the University of Houston. J.M.B acknowledges support from National Science Foundation (Career Award ECCS-1240510) and the Robert A. Welch Foundation (E-1728). 


\section{Notes and References}

1. Mayer, K.M. and Hafner, J.H 2011 Localized surface plasmon resonance sensors Chemical Review 111(6) 3828-3857

2. Haynes, C.L. and Van Duyne R.P 2002 Plasmon Scanned Surface-Enhanced Raman Scattering Excitation Profiles Materials Research Society Symposium $\mathbf{7 2 8}$

3. Stiles, P.L., et al. 2008 Surface Enhanced Raman Spectroscopy Annual Review of Analytical Chemistry 1 601-26

4. Jung, H., et al. 2014 Enhanced photovoltaic properties and long-term stability in plasmonic dyesensitized solar cells via noncorrosive redox mediator ACS Applied Materials \& Interfaces 6 (21) 19191-19200

5. Uddin, A. and Yang, X 2014 Surface plasmonic effects on organic solar cells Journal of Nanoscience and Nanotechnology 14(2) 1099-119

6. Luther, J. M 2011 Localized surface plasmon resonances arising from free carriers in doped quantum dots Nature Materials 10 361-366

7. Kim, K. et al. 2009 Localized surface plasmon resonance detection of layered biointeractions on metallic subwavelength nanogratings Nanotechnology 20(31)

8. Merlen, A. and Lagugne'-Labarthet, F 2014 Imaging the Optical Near Field in Plasmonic Nanostructures Applied Spectroscopy 68(12) 1307-1326

9. Huang, X. X 2010 Interface between Nanophotonics and Biotechnology: How the Near-Field Can Boost Proteomics Based on LSPR Nano Sensor Advanced Materials Research 139-141 1554-1557

10. Law, W. L 2011 Nanostructure materials for biosensing and bioimaging applications Thesis (Ph.D.)-State University of New York at Buffalo, Dissertation Abstracts International 72-04 Section: B, 161

11. Huang, X. and El-Sayed, M. A 2011 Plasmonic photo-thermal therapy (PPTT) Alexandria Journal of Medicine 47(1) 1-9

12. Willets, K.A., and Van Duyne R.P 2007 Localized surface plasmon resonance spectroscopy and sensing Annual Review of Physical Chemistry 58 267-297

13. Petryayeva, E., and Krull, U.J 2011 Localized surface plasmon resonance: nanostructures, bioassays and biosensing-a review Analytica Chimica Acta 706(1) 8-24

14. Lu, X., et al. 2009 Chemical synthesis of novel plasmonic nanoparticles Annual Review of Physical Chemistry 60 167-92

15. Takeda, Y., et al. 2006 Surface Plasmon Resonance of Au Nanoparticles Fabricated by Negative Ion Implantation and Grid Structure toward Plasmonic Applications Optical Review 13(4) 231-234

16. Seo, H., et al. 2012 Formation of silver nanoparticles in silicon by metal vapor vacuum arc ion implantation Nuclear Instruments and Methods in Physics Research Section B: Beam Interactions with Materials and Atoms 292 50-54

17. Wijesundera, D.N., et al. 2013 Ion beam engineered nano silver silicon substrates for surface enhanced Raman spectroscopy Journal of Raman Spectroscopy 44 1014-1017

18. Alexandra, B 2009 Plasmonic components fabrication via nanoimprint Journal of Optics A: Pure and Applied Optics 11(11) 114001

19. Rechberger, W., et al. 2003 Optical properties of two interacting gold nanoparticles Optics Communications 220(1) 137-141

20. Pisarenko, A.N., et al. 2009 Detection of ozone gas using gold nanoislands and surface plasmon resonance Talanta $\mathbf{8 0}$ (2) 777-80

21. Gupta, G., et al. 2009 Absorption spectroscopy of gold nanoisland films: optical and structural characterization Nanotechnology 20(2) 025703 
22. Kosiorek, A., et al. 2005 Fabrication of nanoscale rings, dots, and rods by combining shadow nanosphere lithography and annealed polystyrene nanosphere masks Small 1(4) 439-44

23. Wiley, B.J., et al. 2008 Fabrication of surface plasmon resonators by nanoskiving single-crystalline gold microplates Nano Letters 8(9) 3023-8

24. Xu, Q., et al. 2006 Surface plasmon resonances of free-standing gold nanowires fabricated by nanoskiving Angewandte Chemie International Edition 45(22) 3631-5

25. Atwater, H.A. and Polman, A 2010 Plasmonics for improved photovoltaic devices Nature Materials 9(3) 205-13

26. Pillai, S., et al. 2007 Surface plasmon enhanced silicon solar cells Journal of Applied Physics 101(9) 093105

27. Lim, S.H., et al. 2007 Photocurrent spectroscopy of optical absorption enhancement in silicon photodiodes via scattering from surface plasmon polaritons in gold nanoparticles Journal of Applied Physics 101(10) 104309

28. Kirkpatrick, A 2003 Gas cluster ion beam applications and equipment Nuclear Instruments and Methods in Physics Research Section B: Beam Interactions with Materials and Atoms 206 830-837

29. Lozano, O., et al. 2013 Evolution of nanoripples on silicon by gas cluster-ion irradiation AIP Advances 3(6) 062107

30. Tilakaratne, B.P 2012 Self-Assembled nano-Patterns by Gas Cluster Ion Beam Bombardment Ph.D. Dissertation, University of Houston

31. Toyoda, N., et al. 2005 Nano structure formation by gas cluster ion beam irradiations at oblique incidence Nuclear Instruments \& Methods in Physics Research Section B-Beam Interactions with Materials and Atoms 232 212-216

32. Yamada, I 1991 New horizons in material processing with ICB Proceedings of 14th Symposium on Ion Sources Ion-Assisted Technology, Tokyo, Japan 227-235

33. Yamada, I., et al. 2001 Materials processing by gas cluster ion beams Materials Science \& Engineering R-Reports 34(6) 231-295

34. Chu, W.K., et al. 1998 Smoothing of YBa2Cu3O7- $\delta$ films by ion cluster beam bombardment Applied Physics Letters 72(2) 246-248

35. Yamada, I 1995 A short review of ionized cluster beam technology Nuclear Inst. and Methods in Physics Research, B 99(1-4) 240-243

36. Tilakaratne, B.P., Wijesundera D.N., Chen Q.Y., and Chu W.K 2016 Investigation of self-assembled gold nano-ripple formation during gas cluster ion beam bombardment Submitted. 


\section{Figure Captions}

Figure 1: A schematic of oblique angle gas cluster ion beam (GCIB) irradiation configuration used in fabrication of nano ripple array structures. In this case presented, a $100 \mathrm{~nm}$ thick film of gold on silicon is irradiated with argon GCIB. $\theta$ is the angle of incidence of GCIB irradiation.

Figure 2: (a) SEM top view image of the nano-ripple array on gold surface obtained for GCIB irradiation fluence of $4 \times 10^{16}$ clusters $/ \mathrm{cm}^{2}$, for an incident angle of 60 degrees. (b) SEM image with the substrate tilted 70 degrees for better visualization of the cross-section of the ripples.

Figure 3: Experimental arrangement used for measuring optical diffuse scattering from nano-rippled gold substrates.

Figure 4: (a) Optical scattering spectra of gold nano-ripple array surfaces fabricated by GCIB irradiation at fluence of $1 \times 10^{16}$ clusters $/ \mathrm{cm}^{2}, 2 \times 10^{16}$ clusters $/ \mathrm{cm}^{2}$, and $4 \times 10^{16}$ clusters $/ \mathrm{cm}^{2}$, for a GCIB incident angle of 60 degrees. Spectra clearly show the enhanced scattering peak due to LSPR of the nano-ripples. The insert shows a comparison of spectra for surface component of polarization of light perpendicular and parallel to the length of ripples showing that LSPR occurs when the polarization is perpendicular to the length of ripples. The frequency of resonance can be effectively changed by changing the GCIB irradiation fluence in fabrication. (b) Variation of average ripple height and (c) average ripple periodicity with respect to GCIB fluence, obtained via atomic force microscopy.

Figure 5: (a) (b) (c) and (d) are the AFM images of gold nano-ripples fabricated by ion fluence of $1 \times 10^{15}$ clusters $/ \mathrm{cm}^{2}, 1 \times 10^{16}$ clusters $/ \mathrm{cm}^{2}, 2 \times 10^{16}$ clusters $/ \mathrm{cm}^{2}$ and $4 \times 10^{16}$ clusters $/ \mathrm{cm}^{2}$ respective

Figure 6: The polarization dependence of the plasmonic property of the nano ripples array surface is addressed. (a) Explains the LSPR effect occurs when the incident E field is perpendicular to the ripple array. That is when the wavelength of the incident $\mathrm{E}$ field is larger than the cross section of the ripples. In the scattering spectrum huge peak in the intensity at the resonance frequency is observed. (b) Shows there is no resonance effect when the incident E field is parallel to the ripple pattern. (c) Shows the drop in the intensity as we change the polarization angle from 90 degrees to 0 degrees for the nanostructure of $4 \times 10^{16}$ clusters $/ \mathrm{cm}^{2}$ fluence. 